Review

\title{
Review of Research Status and Development Trends of Wireless Passive LC Resonant Sensors for Harsh Environments
}

\author{
Chen Li ${ }^{1,2}$, Qiulin Tan ${ }^{1,2, *}$, Pinggang Jia ${ }^{2}$, Wendong Zhang ${ }^{1}$, Jun Liu ${ }^{1}$, Chenyang Xue ${ }^{2}$ and \\ Jijun Xiong 1,2,*
}

1 Key Laboratory of Instrumentation Science \& Dynamic Measurement, Ministry of Education, North University of China, Tai Yuan 030051, China; E-Mails: flanklichen@163.com (C.L); wdzhang@nuc.edu.cn (W.Z.); liuj@nuc.edu.cn (J.L.)

2 Science and Technology on Electronic Test \& Measurement Laboratory, North University of China, Tai Yuan 030051, China; E-Mails: pgjia@nuc.edu.cn (P.J.); xuechenyang@nuc.edu.cn (C.X)

* Authors to whom correspondence should be addressed; E-Mails: tanqiulinnuc@163.com (Q.T.); xiongjijun@nuc.edu.cn (J.X.); Tel.: +86-351-3922131 (Q.T.); +86-351-3921882 (J.X.).

Academic Editor: Ha Duong Ngo

Received: 6 April 2015 / Accepted: 1 June 2015 / Published: 4 June 2015

\begin{abstract}
Measurement technology for various key parameters in harsh environments (e.g., high-temperature and biomedical applications) continues to be limited. Wireless passive LC resonant sensors offer long service life and can be suitable for harsh environments because they can transmit signals without battery power or wired connections. Consequently, these devices have become the focus of many current research studies. This paper addresses recent research, key technologies, and practical applications relative to passive LC sensors used to monitor temperature, pressure, humidity, and harmful gases in harsh environments. The advantages and disadvantages of various sensor types are discussed, and prospects and challenges for future development of these sensors are presented.
\end{abstract}

Keywords: passive LC sensor; mutual inductance coupling; noncontact wireless measurement; harsh environments 


\section{Introduction}

Harsh environments can typically be subdivided into high-temperature, biocompatible, corrosive and chemical, etc. In addition, wireless passive sensors used in these harsh-environment applications can be categorized as high-temperature, biocompatible, and humidity and gas sensors (Table 1). Measurements of certain key parameters (e.g., pressure, temperature, and humidity) in harsh environments have become increasingly important, especially pressure measurements in high-temperature environments. Figure 1 shows various harsh-environment sensing applications. Temperature and pressure measurements are critical for system control in high-temperature (such as in aerospace, automotive, aeronautics, and industrial) applications [1-4]. For biomedical applications, real-time pressure monitoring, e.g., in eyes, blood vessels, abdominal aortic aneurysm, or brain, is crucial for diagnosis, disease prevention, and treatment. Further, in some special industrial applications, wireless sensors are required to measure humidity or detect harmful gases. Many sensors have been extensively researched to monitor these key parameters in harsh environments, but their practical use has been limited due to such disadvantages as complex design, or dependence on battery power. Wireless sensing technology has a large potential for measurement in these harsh environments, and the development of wireless measurement technology has attracted significant interests. Typical commercial wireless passive sensors can be divided into surface acoustic wave (SAW), RF identification (RFID), intermodulation sensor, and RF-powered LC sensor. SAW sensors can be used for wireless monitoring in harsh environments. However, the piezoelectric material may limit their applications, and the highest operating frequency is limited to several gigahertz. RFID is mostly used for identification and can be used for telemetry, but the highest operating frequency is limited by the rectified power of the integrated circuit. The intermodulation read-out principle of a passive wireless sensor enables very small implantable health and fitness sensors, which can potentially be used for wireless monitoring (such as strain in bridges and building structure moisture, etc.). However, sensors based on this concept for harsh environments (such as high-temperature, biomedical, and other applications) have not been reported yet [5-7]. Wireless passive LC sensors are composed of inductance and capacitance and do not require wired connections to transmit signals. Thus, they are potential for complex applications in harsh environments. In addition, owing to their lower operating frequency and near-field coupling distance, passive LC sensors can achieve highly energy-efficient transmission and highly efficient data collection in harsh environments. Recently, numerous studies have investigated passive LC sensors to measure pressure, temperature, and humidity in harsh environments. Furthermore, the development of wireless passive measurement technology can address issues (such as unstable ohmic contacts or inadequate packaging) that are particularly problematic in environments involving high temperature, high rotation, sealed enclosures, or biological tissues. 
Table 1. Sensor types, sensor characteristics, and harsh-environment applications.

\begin{tabular}{|c|c|c|}
\hline Category & Characteristics & Typical Applications \\
\hline Biomedical sensors & In vivo & $\begin{array}{l}\text { Pressure monitoring in eyes, blood vessel, and } \\
\text { abdominal aortic aneurysm, etc. }\end{array}$ \\
\hline High-temperature sensors & -55 to $1500^{\circ} \mathrm{C}$ & $\begin{array}{c}\text { Aircrafts, engines, steel process control, and } \\
\text { environmental monitoring }\end{array}$ \\
\hline Humidity and gas sensors & $\begin{array}{l}\text { Resistant to corrosion } \\
\text { and high humidity }\end{array}$ & $\begin{array}{l}\text { Harmful component monitoring in chemical } \\
\text { plants, fuel cells, and packaged foods, etc. }\end{array}$ \\
\hline
\end{tabular}

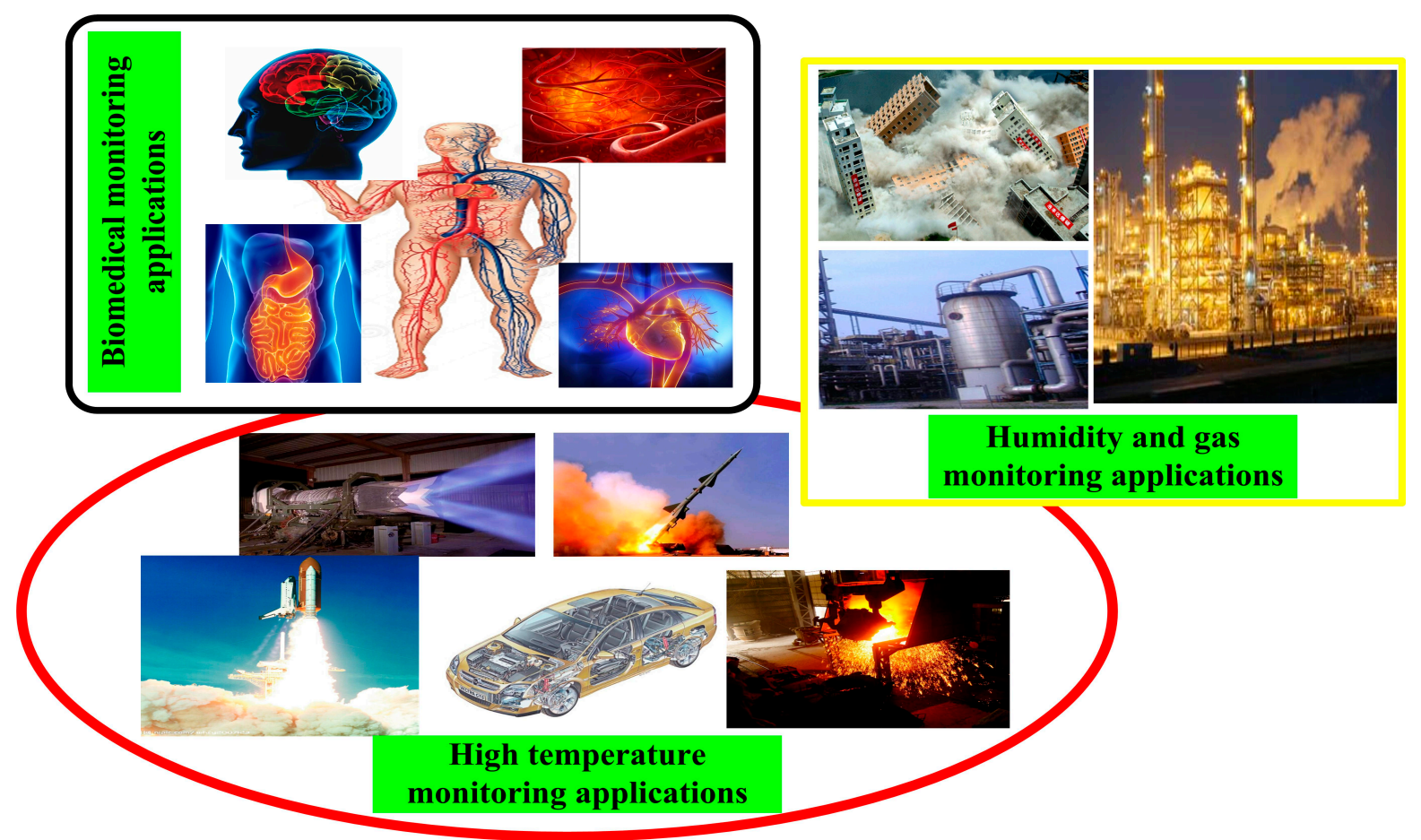

Figure 1. Examples of high-temperature, biomedical, and humidity and gas monitoring applications in harsh environments.

This paper summarizes the wireless measurement principle, model design, and latest research on passive LC sensors applied to monitor pressure, temperature, humidity, and harmful gases. Development trends are also predicted.

\section{Wireless Measurement Principles and Sensor Design Model}

The lumped circuit model of a wireless passive LC sensor is equivalent to an LC circuit, where $\mathrm{C}$ is the sensitivity capacitance (Figure 2). The resonant frequency of a sensor drifts according to variations in the measured parameters (e.g., temperature, pressure, and humidity). When the swept-frequency signal generated by the measurement antenna passes over the resonant frequency of the sensor, the impedance characteristics (such as phase and magnitude) as seen by the antenna can be extracted because of the coupling link between the measurement antenna and the sensor. Thus, wirelessly measuring the resonant frequency of the sensor becomes possible. Figure 2 shows the process of performing wireless sensor measurements: $f_{\mathrm{s}}$ is the sensor resonant frequency, and $f_{\min }$ is the frequency of the lowest point of the phase angle as seen by the measurement antenna [8]. 


$$
f_{\min }=f_{S}\left(1+\frac{k^{2}}{4}+\frac{1}{8 Q^{2}}\right)
$$

where $k$ is the coupling coefficient between the inductance coils of the antenna and the sensor, and $Q$ is the quality factor of the sensor. If $k$ is sufficiently low and $Q$ is sufficiently high, the resonant frequency of the sensor in a harsh environment can be determined by monitoring the variation in $f_{\min }$. Thus, the sensor is an LC resonant circuit with variable capacitance that can wirelessly communicate with an external measurement antenna.

The sensor can be integrated into a measurement system by magnetically coupling it with a loop antenna with inductance $L_{\mathrm{r}}$ so that the magnitude and phase of the sensor impedance can be wirelessly retrieved. Analysis of the overall circuit demonstrates that the impedance can be obtained as follows [9]:

$$
Z_{e q}=\frac{V_{1}}{I_{1}}=j 2 \pi f L_{\mathrm{r}}\left[1+k^{2} \frac{\left(\frac{f}{f_{s}}\right)}{1-\left(\frac{f}{f_{s}}\right)^{2}+\frac{1}{Q_{s}} j \frac{f}{f_{s}}}\right]=F\left(f_{s}\right)
$$

where $V_{1}$ and $I_{1}$ are the voltage and current, respectively, of the measurement antenna, $L_{\mathrm{r}}$ is the inductance of the antenna, and $f$ is the excitation frequency.

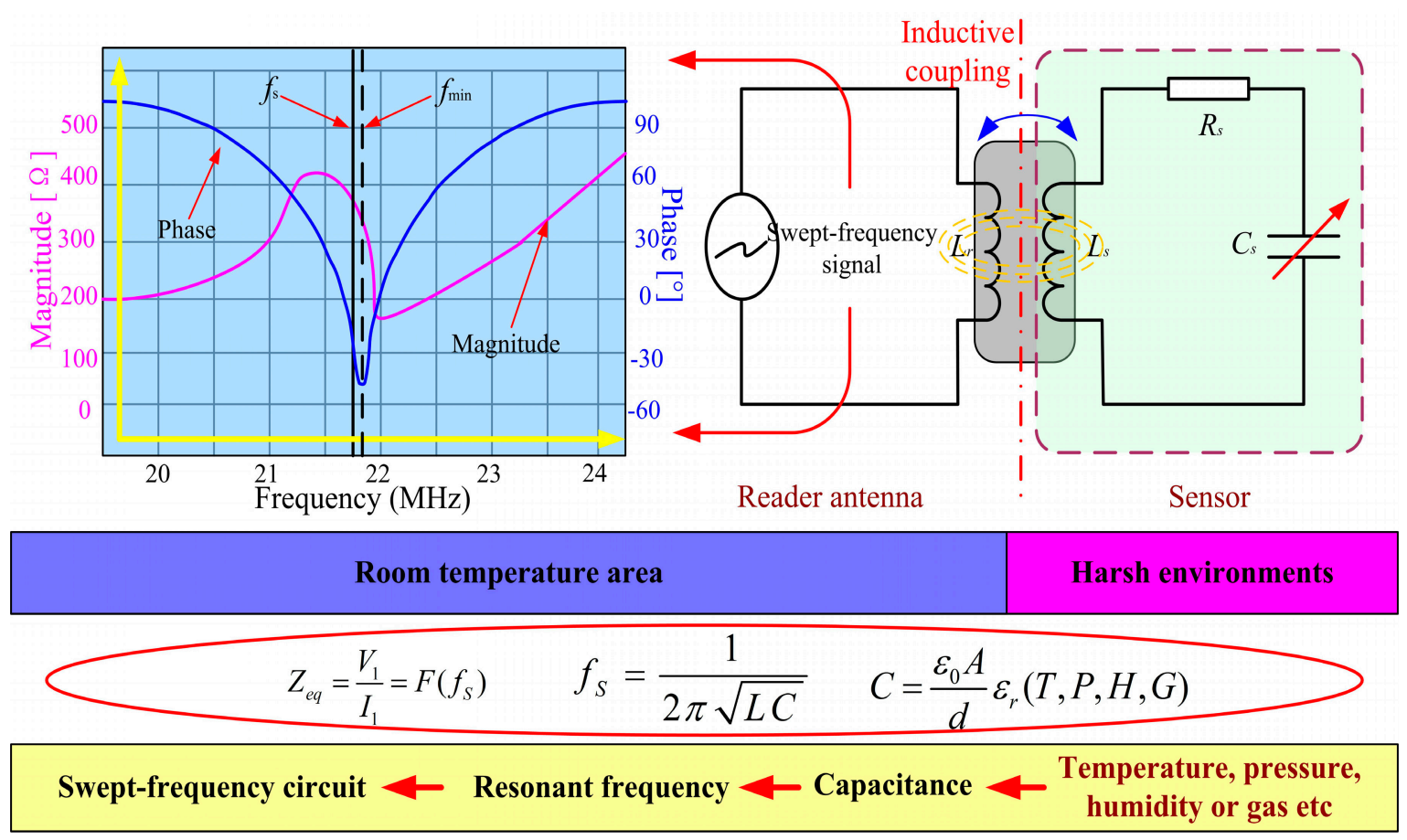

Figure 2. Principles and equations for wireless sensor measurements.

The quality factor of a sensor describes the energy transmission efficiency of a given passive LC resonant sensor. An inductor with higher quality factor is beneficial because it can acquire more power via inductive coupling and can more effectively transmit data to an external receiver. To improve the performance of spiral inductors, designers have evaluated spiral inductors with square, hexagonal, octagonal, and circular cross sections. In 1928, Wheeler H.A. proposed a formula for calculating the approximate inductance of a square planar spiral inductor as follows [10]: 


$$
L \approx 2.34 u_{0} \frac{n^{2} d_{a v g}}{1+2.75 \rho}
$$

where $d_{\text {avg }}=\left(\left(d_{\text {out }}+d_{\text {in }}\right) / 2\right)$ is the average diameter of the inductance coil and $\rho=\left(\left(d_{\text {out }}-d_{\text {in }}\right) /\left(d_{\text {out }}+d_{\text {in }}\right)\right)$ is the fill ratio. $d_{\text {out }}$ is the coil outer diameter, $d_{\text {in }}$ is the coil inner diameter, $u_{0}$ is the permeability of free space, and $n$ is the number of turns of the inductance coil. In 1999, Lee T. proposed an accurate formula to calculate the inductance of a square planar spiral inductor [11].

$$
L=\frac{u_{0} n^{2} d_{\text {avg }} c_{1}}{2}\left(\ln \left(c_{2} / \rho\right)+c_{3} \rho+c_{4} \rho^{2}\right)
$$

where $c_{1}, c_{2}, c_{3}$, and $c_{4}$ are constants determined by the shape of the inductance coil. If the construction area, line width, line thickness, and materials of the inductance coil are given, the quality factor can be improved by improving the ratio of the inner and outer diameters of the inductance coil. The quality factor can be expressed as follows [12]:

$$
Q=\frac{\text { wud }_{\text {out }} h l(1-\alpha)}{4 \pi \rho_{r} s}\left(\ln \frac{1+\alpha}{1-\alpha}+0.2235 \frac{1-\alpha}{1+\alpha}+0.276\right)
$$

where $w, u, h, l, \rho \mathrm{r}$, and $s$ are the angular frequency of the sensor, relative permeability of air, height of the inductance coil, width of the inductance coil, resistivity of the inductance coil, and spacing between adjacent turns of the inductance coil, respectively. $a$ is the ratio of the inner and outer diameters of the inductance coil, which can be improved to achieve a maximum $Q$ value.

\section{Research Status of Wireless Passive LC Sensors for Harsh Environments}

\subsection{High-Temperature Environments}

\subsubsection{High-Temperature Sensors for Pressure Measurements}

For silicon or silicon-on-insulator materials, the mechanical properties easily deteriorate, and the leakage current across the junctions drastically changes with increasing temperature, which limits the operating temperature range of the sensors based on these materials. Ceramic is a good insulator and is chemically stable in high-temperature environments, which enables sensors based on ceramics capable of wide application in high-temperature environments. The fabrication process of high-temperature passive LC ceramic pressure sensors is simple, which includes milling, tape casting, cutting, punching, screen printing, stacking, lamination, and firing. A typical structure of an LC ceramic pressure sensor is shown in Figure 3. In 1999, the team of Professor M. G Allen at the Georgia Institute of Technology pioneered and developed a wireless passive LC pressure sensor based on low-temperature co-fired ceramic (LTCC) technology. Experimental results demonstrated that the average accuracy and sensitivity of this sensor are $24 \mathrm{mbar}$ and $-141 \mathrm{kHz} / \mathrm{bar}$, respectively, at $400{ }^{\circ} \mathrm{C}$ within a pressure range of 0 to 7 bar [13-15]. In 2009, a research team in Novi Sad, Serbia, proposed a better structural model. The improved structural design and the use of LTCC material made this embedded structural sensor suitable for high-temperature and chemically aggressive environments [16,17]. In 2013, researchers at the North University of China, which has been committed to developing wireless, high-temperature passive LC ceramic pressure sensors, introduced a unique screen-printing process involving a 
sacrificial layer of ESL 4900 to prevent deformation of a capacitive embedded cavity during lamination and sintering. This approach improved the flatness of the sensor cavity and led to better performance. The sensitivity of LTCC pressure sensors can reach $-344 \mathrm{kHz} / \mathrm{bar}$ [18], the sensitivity of sensors based on high-temperature cofired ceramic (HTCC) can reach $860 \mathrm{~Hz} / \mathrm{bar}$ [19,20], and sensors based on zircon ceramics can maintain stable operation at $800{ }^{\circ} \mathrm{C}$ for $30 \mathrm{~min}$ [21]. In 2014, researchers at the North University of China proposed improved structural models based on the LTCC, HTCC, and thick-film integrated technologies to improve the performance parameters (e.g., sensitivity, pressure range, operating temperature range, and coupling distance between the sensor and antenna) in harsh environments. Experimental results demonstrated good performance of these improved sensors, namely, sensitivity of up to $13 \mathrm{kHz} / \mathrm{kPa}$, maximum working distance of up to $5 \mathrm{~cm}$, maximum working pressure of up to $60 \mathrm{bar}$, and consistently low repeatability and hysteresis errors [22-26].

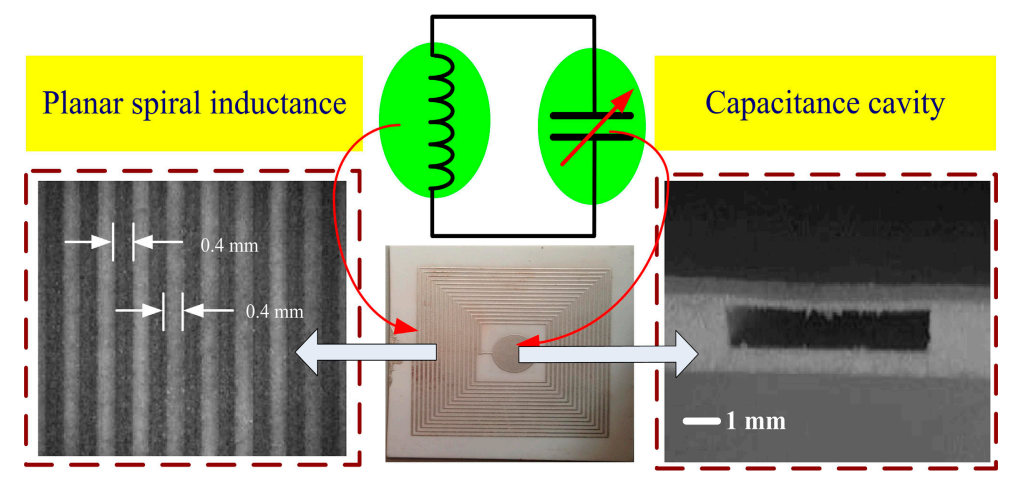

Figure 3. Wireless passive LC ceramic pressure sensor.

\subsubsection{High-Temperature Sensors for Temperature Measurement}

Accurate temperature measurement in harsh environments faces many challenges, but wireless passive LC sensors have become increasingly suitable for these harsh-environment applications. In 2009, J. Yi proposed a wireless passive temperature sensor (Figure 4a) to manufacture the components of a roller bearing. Ferroelectric ceramic was used for the sensor fabrication. With this sensor, the relative dielectric constant increases as the temperature increases. Experimental results demonstrated that the sensitivity of the sensor can be as high as $13 \mathrm{~Hz} /{ }^{\circ} \mathrm{C}$, which satisfies the temperature monitoring requirements for the manufacture of mechanical bearings [27]. In 2014, passive LC temperature sensors based on LTCC or HTCC were proposed. The typical structure of a passive LC temperature sensor is shown in Figure 4b. Initially, a wireless passive temperature sensor intended for harsh environments was realized using the LTCC technology. The dielectric of this sensor is based on ferroelectric ceramic, which exhibits temperature-sensitive permittivity. The sensor sensitivity is approximately $-5.75 \mathrm{kHz} /{ }^{\circ} \mathrm{C}$ from room temperature to $-430{ }^{\circ} \mathrm{C}$ and $-16.67 \mathrm{kHz} /{ }^{\circ} \mathrm{C}$ from 430 to $700{ }^{\circ} \mathrm{C}$ [28]. Subsequently, to develop a sensor that can operate in higher temperature environments, Tan et al. proposed a wireless passive temperature sensor fabricated using multilayer HTCC tapes. The average sensitivity of this sensor, which exhibits good repeatability and reliability, can reach $5.22 \mathrm{kHz} /{ }^{\circ} \mathrm{C}$ with a temperature range from room temperature to $900{ }^{\circ} \mathrm{C}[29]$. 


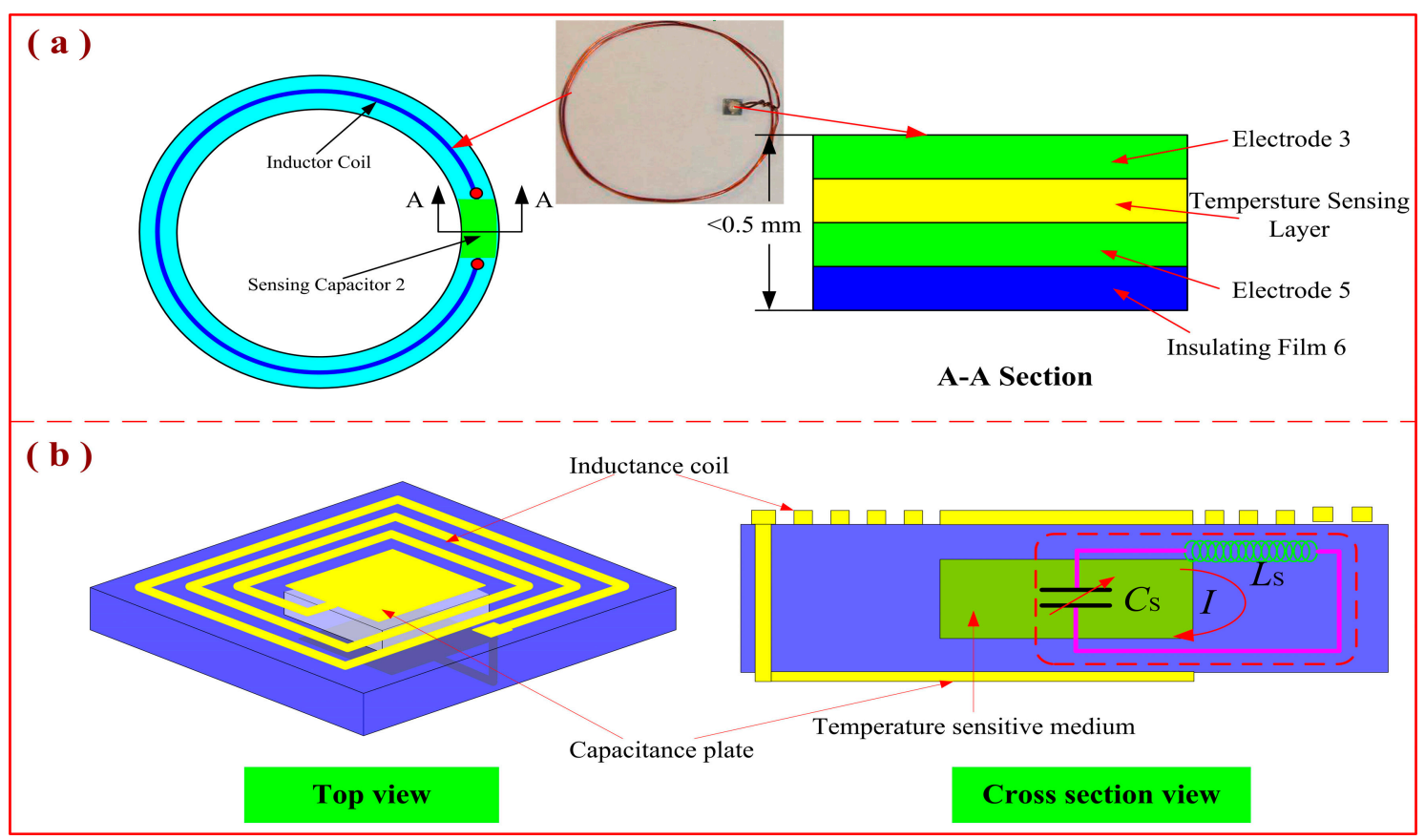

Figure 4. Wireless passive LC temperature sensors.

\subsection{Biomedical Environments}

To achieve pressure monitoring in, e.g., eyes, blood vessels, abdominal aortic aneurysm, or brain, researchers have explored passive wireless biomedical pressure sensors. Numerous studies have investigated pressure sensors for biomedical applications, and the principle of LC resonance coupling is currently attracting intense attention [30-33]. Researchers at the University of Liverpool proposed a passive wireless pressure sensor fabricated using micro-electromechanical systems (MEMS) technology and incorporating a biologically compatible waterproof material. The sensitivity of this sensor can reach $42.85 \mathrm{kHz} / \mathrm{mmHg}$. Professor Y.C. Tai from the California Institute of Technology also demonstrated a wireless passive LC intraocular pressure (IOP) sensor based on parylene materials. The actual response of the sensor was evaluated in the eye of a rabbit. The results indicate that the sensitivity of the sensor can reach $205 \mathrm{kHz} / \mathrm{mmHg}$ with 1-mmHg resolution. Preliminary implementation of this sensor system has already been performed (Figure 5). In 2013, Professor G. Z. Chen proposed a capacitive contact lens sensor for continuous non-invasive IOP monitoring, which could track pressure variations over time with minimal lag. In addition, the IOP sensitivity of the sensor can reach up to $200 \mathrm{ppm} / \mathrm{mmHg}$, and the linearity of the sensor is good, which can be up to 0.997 , obtained in a porcine-eye test. In 2014, Professor J.B. Wang proposed a new differential transduction circuit based on the LC mutual inductance detection mechanism for gastrointestinal pressure monitoring, which is composed of a sensor capsule and a detection unit. The micro-pressure system can realize long-term monitoring stability, and the sensitivity can reach up to $0.2491 \mathrm{kHz} / \mathrm{kPa}$ [34-36]. 


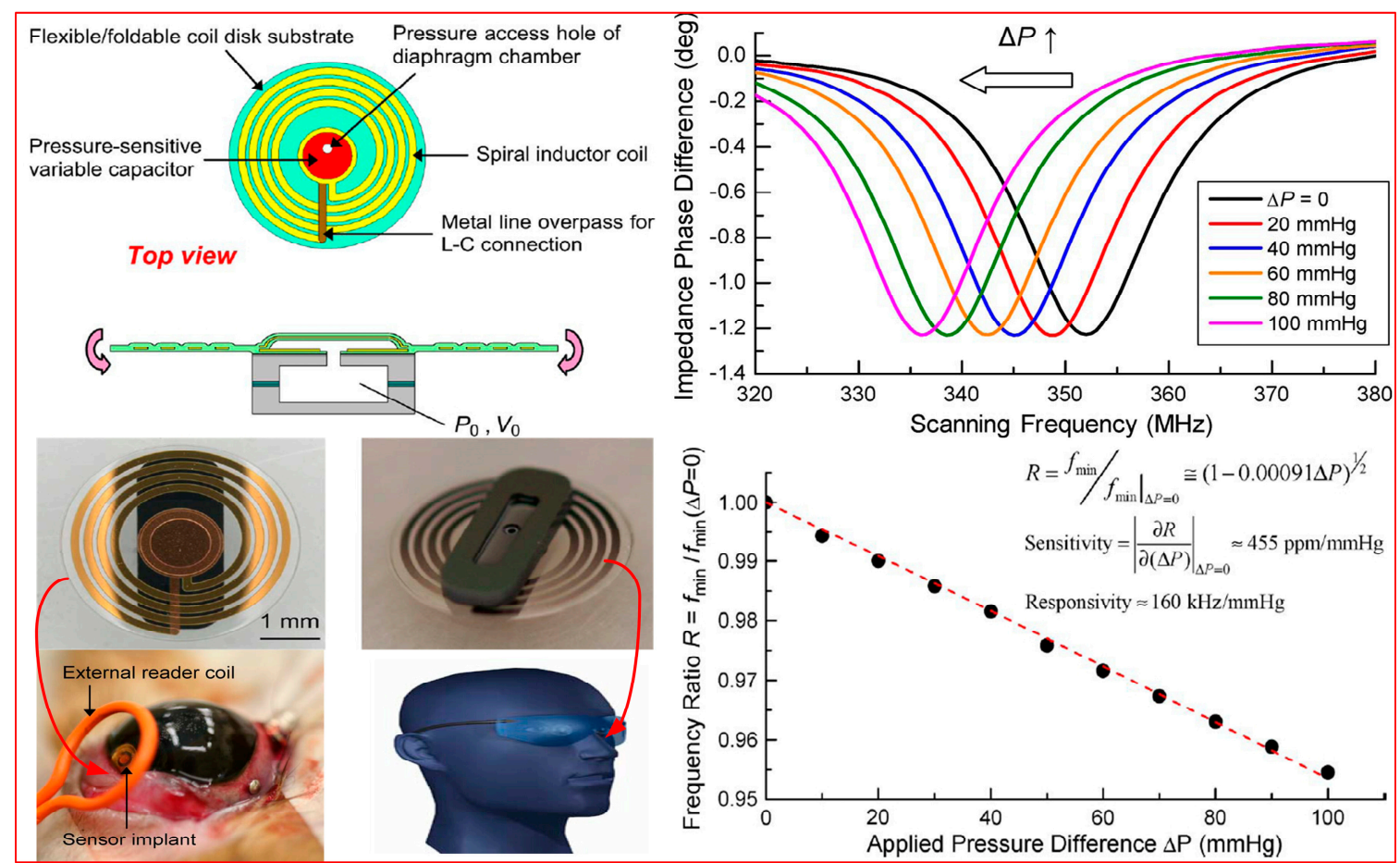

Figure 5. Implantable wireless passive LC pressure sensor for biomedical applications together with the experimental results.

\subsection{Harmful Components of Harsh Environments}

\subsubsection{Humidity Monitoring}

Wireless passive LC sensors offer a highly advantageous ability to provide humidity monitoring in some special environments such as in concrete structures and food packaging. In these sensors, a humidity-sensitive material such as polyimide is deposited onto an interdigital capacitance. When the humidity-sensitive material absorbs moisture from the surrounding environment, the relative dielectric constant of the sensitive material changes, and thus, the interdigital capacitance changes. Consequently, the resonant frequency of the sensor can be determined by an external measurement antenna. An LTCC passive sensor for monitoring the humidity of building materials was proposed by G.M. Stojanovi et al. [37]. LTCC materials were used to fabricate the sensor, and as a result, the sensor exhibited good temperature stability. E.L. Tan et al. proposed a humidity sensor for in situ monitoring of the quality of dry packaged foods such as baked and fried snacks and cereals [38]. Because the dielectric constant of the test samples was much lower than that of water, higher moisture content in the samples caused the capacitance of the sensor to increase, leading to a decrease in the resonant frequency of the sensor. This change in the resonant frequency can be detected by a measurement antenna, and thus, the system can monitor humidity changes inside the package that could lead to degradation in the food quality.

\subsubsection{Gas Monitoring}

In 2002, K.G. Ong at Pennsylvania State University proposed a wireless passive LC gas sensor [39-41]. A multiwalled carbon nanotube was used for the gas-sensitive dielectric material of the sensor, which 
can monitor $\mathrm{CO}$ and $\mathrm{NH}_{3}$ levels. When the $\mathrm{SiO}_{2}$ of the multiwalled carbon nanotube absorbs gases, the dielectric constant and conductivity of the material change according to the gas composition. This change leads to a change in the resonant frequency of the sensor, which is wirelessly detected by an external measurement antenna. This sensor can be used to monitor long-term harmful gas concentrations in a sealed opaque container. Professor Y. Ling from the University of Massachusetts Lowell fabricated a passive LC sensor based on single-walled carbon nanotube materials to monitor $\mathrm{NH}_{3}$ levels. The average sensitivity of this sensor was $0.76 \% / 10^{-6}$ [42].

\section{Development Trends of Passive LC Sensors}

With the development of multifunction sensors, wireless passive LC sensors have also gradually integrated multiple measurement functions. The team of Professor Huang at Southeast University has investigated passive integrated sensors for Internet applications. Figure 6 shows an integrated sensor proposed by the researchers at Southeast University. The sensor is based on MEMS technology. It can simultaneously measure multiple parameters (such as temperature and pressure) and operate at temperatures up to $100{ }^{\circ} \mathrm{C}$ [43]. However, measuring multiple parameters using a single sensor, especially in harsh-environment applications such as high-velocity aeronautical measurements or internal-engine monitoring, suffers from many significant disadvantages. For the multifunction-sensor design, multiple coupling fields are needed to measure multiple parameters. The sensor design is complex compared with that of the signal coupling fields. Fabrication of the sensor faces many challenges in terms of the selection of dielectric materials and fabrication of the sealed cavity. For the measurement of multifunction sensors, because of the mutual inductance among multiple parameters, precise readout of multiple parameters requires a complex decoupling method. In addition, many other challenges exist, including poor adaptability, complex installation, and high cost, etc., and current passive LC sensors exhibit some degree of temperature drift. Therefore, temperature compensation based on a high-temperature testing process is crucial to obtain accurate high-temperature data. Consequently, future development in this field will focus on passive LC sensors that can effectively and simultaneously measure multiple parameters in harsh environments using a single device.

In addition, passive LC sensors can benefit from new materials that provide improved sensitivity and wider operating ranges. The measurement system is also an important area for future development. Passive LC sensors will become even more suitable for harsh-environment applications when combined with smaller and more effective measurement system.

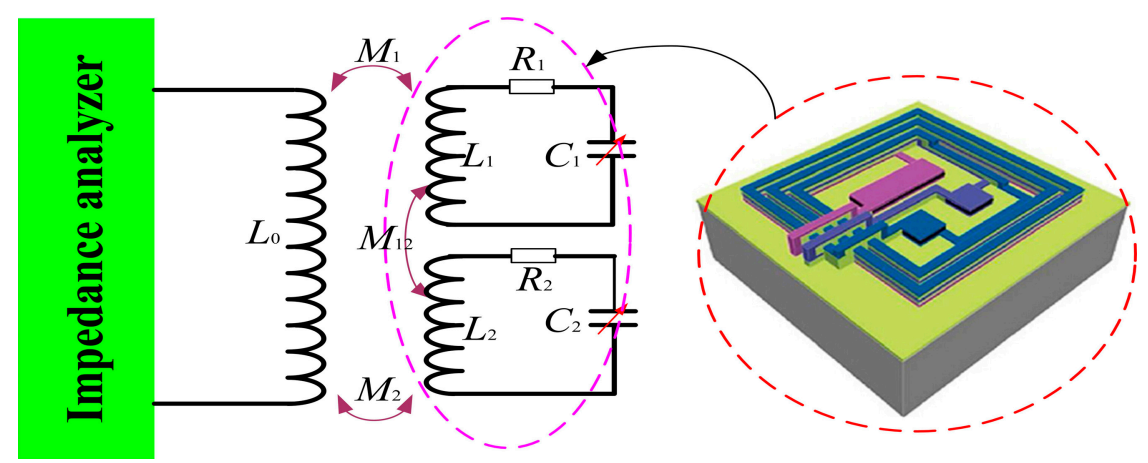

Figure 6. Model of a passive LC sensor capable of simultaneously measuring multiple parameters. 


\section{Conclusions}

Passive LC resonant sensors do not require wired connections or external power supplies, and thus, they can readily be applied to complex measurements in harsh environments. Furthermore, these sensors can realize high-speed data collection and perform highly efficient energy transfer within smaller frequency ranges and working distances. Thus, research into passive LC sensors has found them advantageous for measuring various physical, chemical, and biological parameters. With expanding needs for effective sensors and the development of micro/nano processing technology, we expect that future research will employ passive LC designs in the development of monolithic sensors capable of measuring multiple parameters.

\section{Acknowledgments}

This work was supported by the National Science Fund for Distinguished Young Scholars (No. 51425505), the National Natural Science Foundation of China (No. 61471324) and the Program for the Outstanding Innovative Teams of Higher Learning Institutions of Shanxi, Research Project Supported by Shanxi Scholarship Council of China (2013-077).

\section{Author Contributions}

All works with relation to this paper have been accomplished by all authors' efforts. Chen Li and Qiulin Tan were responsible for the paper's organization and data collection. Pinggang Jia and Wendong Zhang focused on the wireless measurement technology for harsh environments. Chenyang Xue and Jun Liu supervised the work and provided valuable feedback for the development of the present work. At last, every segment relate to this paper is accomplished under the guidance from Jijun Xiong.

\section{Conflicts of Interest}

The authors declare no conflict of interest.

\section{References}

1. Johnson, R.W.; Evans, J.L.; Jacobsen, P.; Thompson, J.R.; Christopher, M. The changing automotive environment: High-temperature electronics. IEEE Trans. Electron. Packag. Manuf. 2004, 27, 164-176.

2. George, T.; Son, K.A.; Powers, R.A.; del Castillo, L.Y.; Okojie, R. Harsh environment microtechnologies for NASA and terrestrial applications. In Proceedings of the IEEE Sensors, Irvine, CA, USA, 30 October 2005; pp. 1253-1258.

3. Werner, M.R.; Fahrner, W.R. Review on materials, microsensors, systems and devices for high-temperature and harsh-environment applications. IEEE Trans. Ind. Electron. 2001, 48, 249-257.

4. Beeby, S.; Ensell, G.; Kraft, M.; White, N. MEMS Mechanical Sensors; Artech House, Inc.: Boston, MA, USA, 2004. 
5. Viikari, V.; Song, J.S.; Seppa, H. Passive wireless sensor platform utilizing a mechanical resonator. IEEE Sens. J. 2013, 13, 1180-1186.

6. Viikari, V.; Seppa, H.; Kim, D.K. Intermodulation read-out principle for passive wireless sensors. Microw. Theory Tech. 2011, 59, 1025-1031.

7. Song, J.S.; Viikari, V.; Pesonen, N.; Marttila, I.; Seppa, H. Optimization of wireless sensor based on intermodulation communication. Microw. Theory Tech. 2013, 61, 3446-3452.

8. Fonseca, M.A.; English, J.M.; Arx, M.; Allen, M.G. Wireless micromachined ceramic pressure sensor for high-temperature applications. J. Microelectromechan. Syst. 2002, 11, 337-343.

9. Nopper, R.; Niekrawietz, R.; Teindl, L. Wireless readout of passive LC sensors. IEEE Trans. Instrum. Meas. 2010, 59, 2450-2457.

10. Wheeler, H.A. Simple inductance formulas for radio coils. Proc. Inst. Radio Eng. 1928, 16, $1398-1400$.

11. Mohan, S.S.; Hershenson, M.D.; Boyd, S.P.; Lee, T.H. Simple accurate expression for planar spiral inductances. IEEE J. Soild-State Circuits 1999, 34, 1419-1424.

12. Zhai, J.; How, T.V.; Hon, B. Design and modeling of a passive wireless pressure sensor. CIRP Ann. Manuf. Technol. 2010, 59, 187-190.

13. English, J.M.; Allen, M.G. Wireless micromachined ceramic pressure sensors. In Proceedings of the Twelfth IEEE Micro-electro-mechanical Systems Conference, Orlando, FL, USA, 21 January 1999; pp. 511-516.

14. Fonseca, M.A. Polymer/Ceramic Wireless MEMS Pressure Sensors for Harsh Environments: High Temperature and Biomedical Applications. Ph.D Thesis, Georgia Institute of Technology, Atalanta, GA, USA, 2007.

15. Birdsell, E.D.; Park, J.; Allen, M.G. Wireless ceramic sensors operating in high temperature environment. In Proceedings of the 40th ALAA/ASME/SAE/ASEE Joint Propulsion Conference, Fort Lauderdale, FL, USA, 11 July 2004.

16. Radosavljevic, G.; Zivanov, L.J.; Smetana, W.; Maric, A.; Unger, M.; Nad, L. A wireless embedded resonant pressure sensor fabricated in the standard LTCC technology. IEEE Sens. J. 2009, 9, 1956-1962.

17. Radosavljevic, G.; Smetana, W.; Mari, A.; Zivanov, L.J.; Unger, M.; Stojanovi, G. Microforce sensor fabricated in the LTCC Technology. In Proceedings of the 27th International Conference on Microelectronics (MIEL 2010), Nis, Serbia, 16-19 May 2010; pp. 16-19.

18. Xiong, J.J.; Li, Y.; Hong, Y.P.; Zhang, B.Z.; Cui, T.H.; Tan, Q.L.; Zheng, S.J.; Liang, T. Wireless LTCC-based capacitive pressure sensor for harsh environment. Sens. Actuators A 2013, 197, 30-37.

19. Xiong, J.; Zheng, S.; Hong, Y.; Li, J.; Wang, Y.; Wang, W.; Tan, Q. Measurement of wireless pressure sensors fabricated in high temperature co-fired ceramic MEMS technology. J. Zhejiang Univ. Sci. C (Comput. Electron.). 2013, 14, 258-263.

20. Zheng, S.J.; Liang, T.; Hong, Y.P.; Xiong, J.J. Fabrication and measurement of wireless pressure-sensitive micro-device based on high temperature cofired ceramics technology. Sens. Rev. 2014, 34, 117-122. 
21. Li, C.; Tan, Q.L.; Xiong, J.J.; Jia, P.G.; Hong, Y.P.; Ren, Z.; Luo, T.; Liu, J.; Xue, C.Y.; Zhang, W.D. A noncontact wireless passive radio frequency (RF) resonant pressure sensor with optimized design for applications in high-temperature environment. Meas. Sci. Technol. 2014, 25, 075101.

22. Li, C.; Tan, Q.L.; Zhang, W.D.; Xue, C.Y.; Li, Y.Z.; Xiong, J.J. Microfabrication of a novel ceramic pressure sensor with high sensitivity based on low-temperature co-fired ceramic (LTCC) technology. Micromachines 2014, 5, 396-407.

23. Tan, Q.L.; Kang, H.; Xiong, J.J.; Qin, L.; Zhang, W.D.; Li, C.; Ding, L.Q.; Zhang, X.S.; Yang, M.L. A wireless passive pressure microsensor fabricated in htcc mems technology for harsh environments. Sensors 2013, 13, 9897-9908.

24. Tan, Q.L.; Qin, L.; Kang, H.; Xiong, J.J.; Zhang, W.D.; Luo, T.; Li, C.; Ding, L.Q.; Zhang, X.S.; Yang, M.L. Zirconia ceramics applied in a pressure-sensitive device fabricated using HTCC technology. Sens. Mater. 2014, 1, 19-30.

25. Luo, T.; Tan, Q.L.; Ding, L.Q.; Wei, T.Y.; Li, C.; Xue, C.Y.; Xiong, J.J. A passive pressure sensor fabricated by post-fire metallization on zirconia ceramic for high-temperature applications Micromachines, 2014, 5, 814-824.

26. Tan, Q.L.; Yang, M.L.; Luo, T.; Liu, W.; Li, C.; Xue, C.Y.; Liu, J.; Zhang, W.D.; Xiong, J.J. A novel interdigital capacitor pressure sensor based on LTCC technology. J. Sens. 2014, 2014, doi:10. 1155/2014/431503.

27. Wang, Y.; Jia, Y.; Chen, Q.S.; Wang, Y.Y. A passive wireless temperature sensor for harsh environment applications. Sensors 2008, 8, 7982-7995.

28. Tan, Q.L.; Luo, T.; Xiong, J.J.; Kang, H.; Ji, X.X.; Zhang, Y.; Yang, M.L.; Wang, X.L.; Xue, C.Y.; Liu, J.; Zhang, W.D. A harsh environment-oriented wireless passive temperature sensor realized by LTCC technology. Sensors 2014, 14, 4154-4166.

29. Tan, Q.L.; Ren, Z.; Cai, T.; Li, C.; Zheng, T.L.; Li, S.N., Xiong, J.J. Wireless passive temperature sensor realized on multilayer HTCC tapes for harsh environment. Available online: http://www.hindawi.com/journals/js/aa/124058 (accessed on September 2014 ).

30. Chen, P.J.; Rodger, D.C.; Saati, S.; Humayun, M.S.; Tai, Y.C. Microfabricated implantable parylene-based wireless passive intraocular pressure sensors. J. Microelectromec. Syst. 2008, 17, $1342-1351$.

31. Chen, P.J.; Saati, S.; Varma, R.; Humayun, M.S.; Tai, Y.C. Wireless intraocular pressure sensing using microfabricated minimally invasive flexible-coiled LC sensor implant. J. Microelectromec. Syst. 2010, 19, 721-734.

32. Chitnis, G.; Maleki, T.; Samuels, B.; Cantor, L.B.; Ziaie, B. A minimally invasive implantable wireless pressure sensor for continuous $1 \mathrm{OP}$ monitoring. IEEE Trans. Biomed. Eng. 2013, 60, 250-256.

33. Xue, N.; Chang, S.P.; Lee, J.B. A SU-8 based microfabricated implantable inductively coupled passive RF wireless intraocular pressure sensor. J. Microelectromec. Syst. 2012, 21, 1338-1364.

34. Shi, Q.; Wang, J.B.; Chen, D.; Chen, J.; Li, J.; Bao, K. In vitro and In vivo characterization of wireless and passive micro system enabling gastrointestinal pressure monitoring. Biomed. Microdevices 2014, 16, 859-868.

35. Chen, G.Z.; Chan, L.S.; Lam, D. Capacitive contact lens sensor for continuous non-invasive intraocular pressure monitoring. Sens. Actuators A. 2013, 203, 112-118. 
36. Bao, K.K.; Chen, D.Y.; Shi, Q.; Liu, L.J.; Chen, J.; Li, J.; Wang, J.B. A readout circuit for wireless passive LC sensor and its application for gastrointestinal monitoring. Meas. Sci. Technol. 2014, 25, 85104-85111.

37. Stojanovic, G.; Radovanovic, M.; Malesev, M.; Radonjanin, V. Monitoring of water content in building materials using a wireless passive sensor. Sensors 2010, 10, 4270-4280.

38. Tan, E.L.; Ng, W.N.; Shao, R.Y.; Pereles, B.D.; Ong, K.G. A wireless passive sensor for quantifying packaged food quality. Sensors 2007, 7, 1747-1756.

39. Ong, J.B.; You, Z.P.; Mills-Beale, J.; Tan, E.L.; Pereles, B.D.; Ong, K.G. A wireless passive embedded sensor for real-time monitoring of water content in civil engineering material. IEEE Sens. J. 2008, 8, 2053-2058.

40. Ong, K.G.; Zeng, K.; Grimes, C.A. A wireless, passive carbon nanotube-based gas sensor. IEEE Sens. J. 2002, 2, 82-88.

41. Ong, K.G.; Zeng, K.; Grimes, C.A. A carbon nanotube-based sensor for $\mathrm{CO}_{2}$ monitoring. Sensors 2001, 1, 193-205.

42. Ling, Y.F.; Zhang, H.; Gu, G.R.; Lu, X.J.; Kayastha, V.; Jones, C.S.; Shih, W.-S.; Jazon, D.C. A printable CNT-based FM passive wireless sensor tag on a flexible substrate with enhanced sensitivity. IEEE Sens. J. 2013, 2, 2546-2550.

43. Zhang, C.; Hang, J.Q.; Hang, Q.A. Design of LC-type passive wireless multi-parameter sensor. In Proceedings of the Nano/Micro Engineered and Molecular Systems (NEMS), Suzhou, China, 7-10 April 2013; pp. 256-259.

(C) 2015 by the authors; licensee MDPI, Basel, Switzerland. This article is an open access article distributed under the terms and conditions of the Creative Commons Attribution license (http://creativecommons.org/licenses/by/4.0/). 